\title{
Simulador de Ruído de Cavitação de Embarcações utilizando uma Rede Neural Realimentada
}

\author{
Anderson Damacena Santos, Elio P. Sarno Filho, \\ Eduardo F. Simas Filho e Antônio C. L. Fernandes Jr. \\ Program in Electrical Engineering \\ Federal University of Bahia \\ Salvador, Brasil \\ (anderson.damacena,elio.pithon,antonio.lopes)@ufba.br
}

\author{
José M. de Seixas \\ Signal Processing Laboratory \\ Federal University of Rio de Janeiro \\ Rio de Janeiro, Brasil \\ seixas@lps.ufrj.br
}

\begin{abstract}
Resumo-Sistemas de sonar passivo são utilizados tanto em aplicações civis quanto militares, para tarefas como monitoramento do ambiente submarino e detecção de animais e embarcações. A aquisição de sinais acústicos submarinos requer um complexo sistema de medição e envolve custos consideráveis para instalação e manutenção. Aliado a isso, a grande variedade de classes de embarcação e condições climáticas aumenta consideravelmente a diversidade destes tipos de sinais. Neste contexto, esse trabalho propõe-se a desenvolver um simulador de sinais acústicos submarinos que combina informações da cavitação de embarcações com ruído ambiente a partir de um sistema com um bloco fenomenológico e um bloco baseado em aprendizado de máquinas. $O$ primeiro produz a combinação de ruído de cavitação de embarcações com ruído ambiente a partir da descrição dos fenômenos físicos utilizando modelos matemáticos. A seguir, uma rede neural realimentada (estrutura NARX) é utilizada para produzir um mapeamento que aproxima o resultado do simulador do comportamento de sinais experimentais. $O$ simulador proposto foi capaz de produzir sinais com grande fidedignidade a sinais reais.
\end{abstract}

Index Terms-Rede Neural, Rede NARX, Simulador, Sonar Passivo

\section{INTRODUÇÃO}

Nas últimas décadas houve um aumento considerável na utilização de sistemas sonar (Sound navigation and ranging) no âmbito submarino [1]. É possível encontrar aplicações em diversas áreas como, por exemplo, detecção e monitoramento de peixes e animais mamíferos usando sonar de imagem [2], detecção de alvos em ambiente subaquático [3] e detecções de danos em estruturas subaquáticas [4] .

Sistemas sonar submarinos são classificados em dois tipos, ativos e passivos [5]. Sonares ativos têm como característica a emissão de um pulso que, ao refletir em algum obstáculo, retorna para a fonte de origem, permitindo assim a detecção, localização e classificação do alvo. Sonares ativos comumente são utilizados em aplicações civis. O sonar passivo é voltado para a detecção e monitoramento de um alvo, a principal característica desse tipo é a não emissão de sinal. Para a deteç̧ão do alvo de interesse o mesmo se aproveita do fato de que o alvo se comporta como uma fonte emissora de sinais acústicos, sinais esses que irão se propagar pelo meio aquático, chegando nos receptores do sonar. O Sonar passivo é fundamental para operações de submarinos militares.
Um dos problemas encontrados ao trabalhar com sonar passivo é a dificuldade de obtenção de dados experimentais. Devido à escassez de dados relacionados à ruídos acústicos submarinos, usualmente pesquisadores precisam criar seu próprio banco de dados, fazendo com que o investimento seja dispendioso, pois, é necessário empregar diversas pessoas, equipamentos e a instalação desses equipamentos. Associado a isso há dificuldade de garantir a condição ambiental no momento da aquisição dos dados.

Outro problema identificado, ao se trabalhar com sonar passivo é que, em condições reais, o sinal de interesse está contaminado por diversas fontes de ruído, tanto ambientais, quanto irradiadas por outras fontes. O ruído ambiente é proveniente de fontes naturais (ruído da chuva, estado do mar, fontes biológicas) [6] e fontes antropogênicas (ruído de tráfego marítimo e exploração de gás e óleo) [7]. O ruído irradiado é proveniente da vibração das máquinas e do equipamento de propulsão das embarcações [5]. Muitas vezes o ruído tem espectro de frequência similar ao sinal produzido pelo alvo de interesse. Uma forma de contornar essa limitação é a utilização de técnicas de processamento de sinais com o objetivo de realizar a separação do sinal de interesse dos ruídos [8].

Uma das técnicas de processamento de sinais voltadas para o sonar passivo é a análise DEMON (Demodulation of envelope modulation on noise), que é uma analisador espectral de banda estreita que trabalha sobre o ruído de cavitação [9], ruído gerado pela rotação do hélice da embarcação. A partir da análise do sinal acústico irradiado das embarcações é possível determinar a velocidade de rotação do eixo do hélice e a quantidade de pás da embarcação de interesse [10], permitindo, assim, a localização e classificação das embarcações.

O processamento para extração de informações é uma parte importante em um sistema sonar. Usualmente feita de forma automática, os módulos de processamento digital ajudam o operador sonar a tomar decisões relacionadas à caracterização da embarcação. Além das informações fornecidas pelos módulos de processamento, o operador sonar pode realizar análises através do áudio do sinal, essa técnica é usada pelo operador com o intuito de auxiliar a identificação das embarcações e para estimar a sua velocidade [11] [12].

Neste contexto, devido à dificuldade de obtenção de dados 
experimentais relacionados a ruídos acústicos submarinos, o presente artigo propõe desenvolver um modelo baseado em conceitos estatísticos e de aprendizado de máquinas. O objetivo é desenvolver um simulador de diferentes tipos de ruídos irradiados de embarcações (ruído de cavitação) em diferentes condições de operação, permitindo assim que a comunidade científica possa adquirir dados, de forma sintética, para o desenvolvimento de soluções em diferentes situações como, por exemplo, validação de técnicas de processamento de sinais de sonar passivo. A Fig. 1 apresenta o diagrama de blocos do modelo proposto, o sistema é constituído por um simulador fenomenológico e uma rede neural.

O simulador fenomenológico é composto por dois módulos de simulação distintos, onde cada módulo é responsável por gerar um determinado tipo de ruído acústico submarino. O sistema tem como intuito gerar sinais simulados de ruído ambiente (ruído estado do mar e chuva) e ruído irradiado (cavitação), onde será possível determinar certas características do sinal a ser gerado como, por exemplo, tipo de ruído ambiente e sua intensidade, e características da embarcação.

Após a geração do ruído, o sinal proveniente do simulador fenomenológico será utilizado como entrada para a rede neural NARX (nonlinear autoregressive network with exogenous inputs). O intuito é utilizar a rede NARX para incorporar as características não-lineares presentes nos sinais experimentais, permitindo assim que o simulador de ruídos acústicos submarinos gere sinais mais realistas.

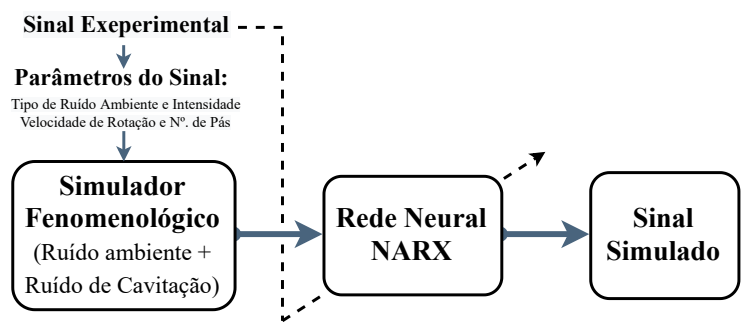

Figura 1: Diagrama de blocos simplificado do simulador acústico.

\section{FundamentaÇão TeÓRICA}

\section{A. Acústica Submarina}

No ambiente acústico submarino existem diversas fontes de ruídos, que podem ser classificadas em duas categorias: ruído irradiado e ruído ambiente [5]. A Fig. 2 demonstra alguns exemplos de ruídos submarinos.

Como o próprio nome diz, o ruído ambiente é proveniente de fontes naturais presentes no ambiente no qual o sonar está localizado. Entre as principais fontes de ruído acústico ambiente, pode-se destacar: estado do mar e chuva [1].

O ruído estado do mar é um ruído proveniente da incidência do vento na superfície do oceano e da quebra das ondas [5], tendo comportamento espectral passa-baixa, ver Fig. 3a. O ruído pode ser classificado em 7 níveis de intensidade, cada um deles está associado à altura das ondas e velocidade do vento.

Já o ruído da chuva é gerado pelo impacto das gotas da chuva na superfície do oceano [5], com comportamento similar

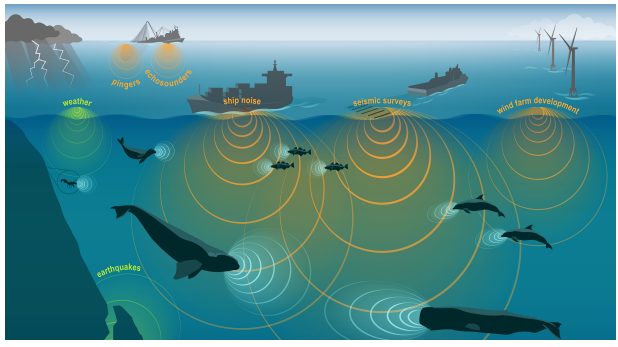

Figura 2: Exemplos de ruídos acústico submarino, adaptado de [13].

a um passa-faixa não simétrico, ver Fig. 3b. O ruído da chuva pode ser classificado em quatro níveis de intensidade, cada nível está relacionado à taxa de precipitação da chuva.

É importante ressaltar que o ruído ambiente é, em geral, prejudicial ao sistema sonar pois pode prejudicar a detecção dos sinais de interesse [14] [15].
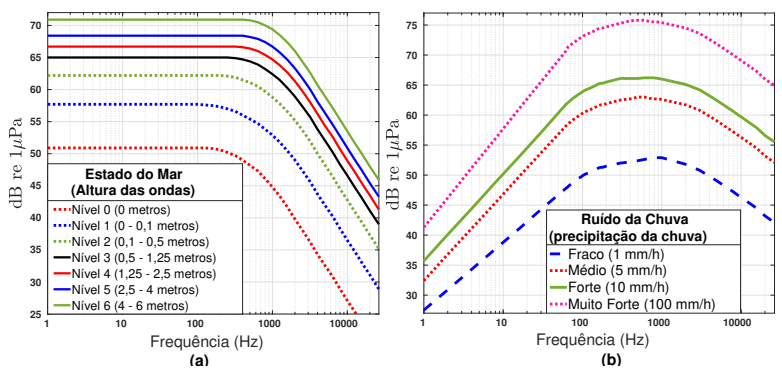

Figura 3: Ruído Ambiente: (a) estado do mar (b) chuva.

O ruído irradiado é proveniente de fontes artificiais, como, por exemplo, submarinos, navios e plataformas para extração de petróleo. Entre os ruídos, pode-se destacar o ruído de cavitação do hélice.

O ruído de cavitação é um ruído proveniente da rotação do hélice da embarcação. Quando o hélice está em funcionamento, ocorre uma redução de pressão, fazendo com que o líquido ao redor evapore formando bolhas de diferentes tamanhos, que irão explodir e implodir, gerando assim um ruído característico. O ruído de cavitação é um sinal modulado em amplitude formado por um sinal de banda estreita (tons espectrais) e banda larga (espectro contínuo).

O ruído de banda estreita é um sinal composto por tons espectrais. Situado abaixo de $1 \mathrm{kHz}$, o ruído está relacionado às características da embarcação, onde, a partir da análise espectral do ruído, é possível estimar algumas características, como, por exemplo, a velocidade de rotação do hélice e a quantidades de pás.

Por sua vez, o ruído de espectro contínuo é gerado a partir do colapso das bolhas de ar formadas a partir da rotação do hélice da embarcação. A banda de frequência do ruído de espectro contínuo vai desde $1 \mathrm{~Hz}$ a $20 \mathrm{kHz}$, tendo amplitude máxima em torno de $100 \mathrm{~Hz}$. Para a banda de frequência de 1 a $100 \mathrm{~Hz}$, o espectro tem um acréscimo entre 6dB/oitava e $12 \mathrm{~dB} /$ oitava. Já para a banda de frequência superior a $100 \mathrm{~Hz}$, 
o espectro de frequência tem um declive de $-6 \mathrm{~dB} /$ oitava. O ruído de banda larga tem comportamento gaussiano colorido.

\section{B. Análise DEMON}

A análise DEMON é um analisador de banda estreita que trabalha sobre o ruído de cavitação. O intuito é, a partir da análise do sinal acústico irradiado, obter informações relacionadas à velocidade de rotação do eixo do hélice e o número de pás da embarcação. A Fig. 4 apresenta um exemplo da análise Demon de uma embarcação hipotética. $\mathrm{O}$ número de harmônicos está relacionado à quantidade de pás do hélice, enquanto a frequência de rotação do eixo do hélice é dada pelo primeiro harmônico do espectro.
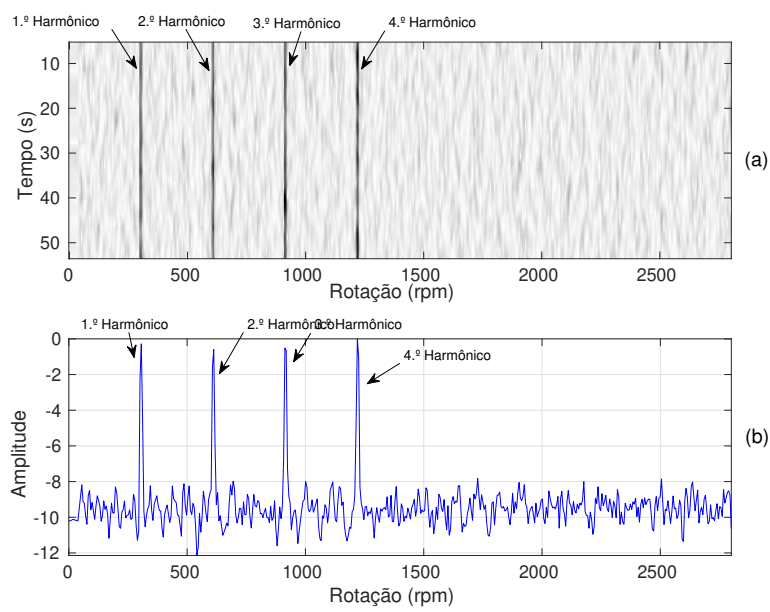

Figura 4: Análise Demon: (a) demograma, (b) espectro médio.

A Fig. 5 mostra o diagrama de blocos da análise DEMON. Inicialmente, o ruído é filtrado utilizando um filtro passa faixa (FPF) com o objetivo de limitar o sinal à banda de frequência mais característica do ruído de cavitação. Uma vez que a frequência de amostragem do sinal é maior que a banda de frequência preservada pelo FPF, após a filtragem é feita uma subamostragem do sinal. Em seguida, o sinal é demodulado para desfazer a modulação em amplitude que ocorre no processo de geração do ruído de cavitação. Com o intuito de obter as informações referentes à velocidade da embarcação, é aplicada a Transformada de Fourier de tempo curto (STFT - Short-time Fourier transform), obtendo assim o espectro de frequência do sinal demodulado. Após a STFT, o sinal é normalizado e apresentado.

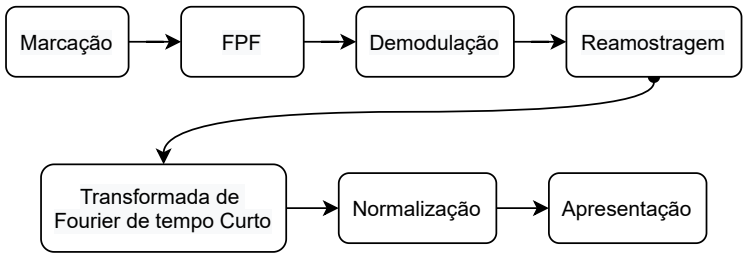

Figura 5: Diagrama de blocos da análise DEMON, Elaborada pelo autor.

\section{Rede Neural NARX}

Com o crescimento das pesquisas relacionadas às redes neurais artificiais novas arquiteturas foram desenvolvidas e uma arquitetura que se destaca são as redes recorrentes (recurrent neural network - RNN). As redes recorrentes se distinguem das demais redes neurais pelo fato de ter pelo menos um laço de realimentação [16]. As RNN são voltadas para o reconhecimento de padrões em sequências de dados. Por considerar o tempo e a sequência do sinal a arquitetura tornase viável para trabalho com processos estocásticos, tanto para sistemas de tempo contínuo quanto para tempo discreto [17].

A arquitetura NARX (Nonlinear autoregressive with exogenous inputs) é uma rede neural recorrente dinâmica comumente usada na modelagem de processos estocásticos [18]. Baseada em uma rede perceptron multicamadas, o modelo NARX possui múltiplas entradas e realimentação de sua saída para a entrada, ver Fig. 6. Tanto a entrada quanto a saída realimenta sofrem atrasos na composição do vetor de entrada.

Considerando a Fig. 6, a rede NARX é descrita por (1) onde $x[n]$ e $y[n]$ são, respectivamente, a entrada e a saída do sistema em intervalos de tempo discreto $n, n_{i} \geq 1 \mathrm{e}$ $n_{j} \geq 1$ são os atrasos de unidade de tempo de entrada e saída, respectivamente e $\varphi$ é a função de entrada-saída da rede.

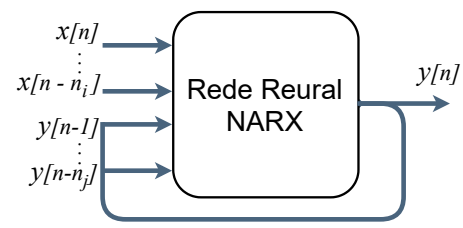

Figura 6: Rede recorrente NARX, Elaborada pelo autor.

$$
y[n]=\varphi\left[y[n-1], \ldots, y\left[n-n_{j}\right], x[n], \ldots, x\left[n-n_{i}\right]\right]
$$

\section{Simulador Proposto}

Para a modelagem dos ruídos ambiente estado do mar e chuva, admitindo que os mesmos tenham distribuição Gaussiana [5], adotou-se modelo proposto na Fig. 7, onde $x[n]$ é um ruído gaussiano branco, $h[n]$ a resposta ao impulso do sistema linear e invariante no tempo (LIT) e $y[n]$ ruído gaussiano colorido.

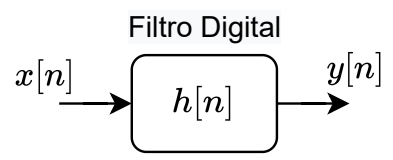

Figura 7: Diagrama de blocos para o sistema proposto.

O modelo proposto tem como finalidade gerar em sua saída um sinal com densidade espectral de potência desejada (PSD - power spectral density). Uma vez que a PSD de um ruído gaussiano branco é constante para toda banda de frequência, é possível obter um ruído gaussiano colorido com PSD específica fazendo com que o ruído branco seja filtrado por um filtro digital linear. 
É mostrado em (2) que a PSD da saída de um sistema LTI, $S_{y}\left(e^{j \omega}\right)$, pode ser obtida a partir da multiplicação da PSD do sinal de entrada, $S_{x}\left(e^{j \omega}\right)$, pelo módulo da resposta em frequência do filtro, $\left|H\left(e^{j \omega}\right)\right|^{2}$. O sistema tem como base a característica de que, em uma transformação linear de um processo dito gaussiano, o resultado produzirá uma saída Gaussiana [19].

$$
S_{y}\left(e^{j \omega}\right)=S_{x}\left(e^{j \omega}\right)\left|H\left(e^{j \omega}\right)\right|^{2}
$$

A partir de (2), considerando que a PSD de saída, $S_{y}\left(e^{j \omega}\right)$, é conhecida e fazendo com que $S_{x}\left(e^{j \omega}\right)$ constante para toda banda de frequência $\omega$ de interesse, pois $x[n]$ é um ruído branco de banda limitada, é possível determinar a magnitude da resposta em frequência para o filtro $\left|H\left(e^{j \omega}\right)\right|$ a partir de (3).

$$
\left|H\left(e^{j \omega}\right)\right|=\sqrt{\frac{\left|S_{y}\left(e^{j \omega}\right)\right|}{\left|S_{x}\left(e^{j \omega}\right)\right|}}=\sqrt{\left|S_{y}\left(e^{j \omega}\right)\right|}
$$

Para estimar $\left|H\left(e^{j \omega}\right)\right|$, foram utilizados os pares $\left|S_{y}\left(e^{j \omega_{i}}\right)\right|$ e $\omega_{i}$ obtidos de [5]. Uma vez que $S_{x}\left(e^{j \omega}\right)$ de um ruído branco é constante para toda banda de frequência de interesse, sem perda de generalidade, neste trabalho, foi adotado $\left|S_{x}\left(e^{j \omega}\right)\right|=1$.

Já o ruído de cavitação, de acordo com [5] é um ruído modulado em amplitude, definido por (4) onde, $S[n]$ é um ruído de banda larga, $A[n]$ é um ruído de banda estreita e $\alpha$ o índice de modulação [1], a Fig. 8 apresenta o diagrama de blocos da modulação em amplitude do ruído.

$$
G[n]=[1+\alpha A[n]] S[n] .
$$

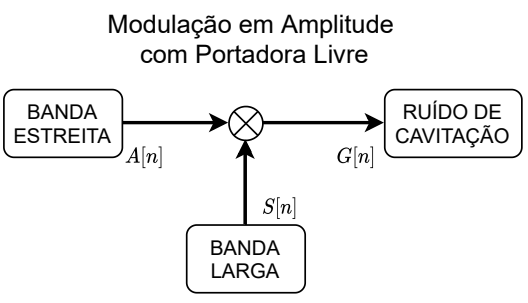

Figura 8: Diagrama de blocos da modulação em amplitude.

Para a obtenção do ruído de banda larga, adotando o modelo da Fig. 7, onde $y[n]=S[n]$, um ruído gaussiano branco $x[n]$ será filtrado por um filtro $h[n]$, gerando na saída do sistema o ruído de banda larga $S[n]$. Para estimar $\left|H\left(e^{j \omega}\right)\right|$ da resposta em frequência do filtro, foram utilizadas as informações espectrais do ruído de banda larga apresentada em [20].

$\mathrm{O}$ ruído de banda estreita é composto a partir de tons espectrais de frequência situados abaixo de $1 \mathrm{kHz}$, ver (5), onde $f_{s h}$ é a frequência de rotação do eixo, $H$ é o número de harmônicos (número de pás) e $A_{n}$ é a amplitude de cada harmônico.

$$
A[n]=\sum_{h=1}^{H} A_{n} \cos \left(2 \pi h f_{s h} n\right)
$$

Após a modelagem do simulador fenomenológico, foi implementa a rede NARX. O ruído gerado pelo simulador fenomenológico será usado como entrada para a rede. O diagrama de blocos do sistema é apresentado na Fig. 9.

Os dados de entrada são compostos por um conjunto de 10 ruídos de cavitação sintéticos (gerados pelo simulador fenomenológico), $x_{n}[n]$. Já para os dados de referência foi utilizado o ruído experimental do banco de dados da Espanha [21].

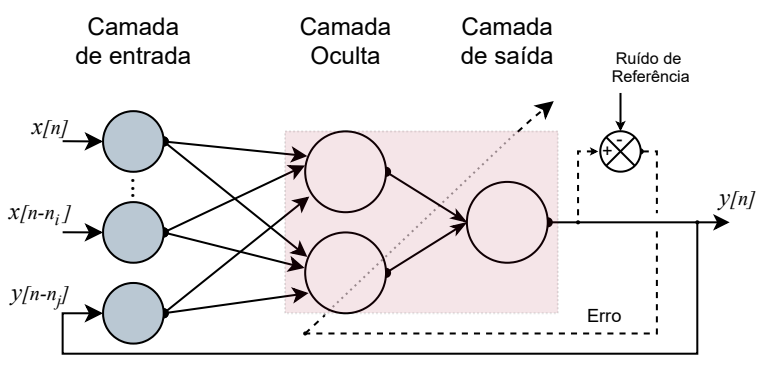

Figura 9: Diagrama de blocos da rede NARX

Para definição dos parâmetros da rede NARX foi adotado o procedimento empírico, onde, após uma série de simulações (variando a quantidade de neurônios na camada oculta e a quantidade de atrasos) foi possível encontrar a configuração que fornecesse o melhor desempenho da rede.

A Tabela I apresenta os parâmetros da rede neural adotada no trabalho. A rede implementada é composta por uma camada oculta, os pesos são inicializados de forma aleatória, conforme o método proposto por Nguyen e Widrow [22]. Como critério de parada, a rede foi configurada para interromper o treinamento caso, após seis iterações não consiga diminuir o erro de validação. Para o treinamento, houve uma variação do número de neurônios da camada oculta (1:10), com o intuito de encontrar a configuração que gerou o melhor resultado.

Para o processo de aprendizagem da rede, o conjunto de dados de entrada foi dividido da seguinte forma: $70 \%$ dos sinais foram direcionados para o treinamento da rede, $15 \%$ para validação e $15 \%$ para teste, com uma função de ativação sigmóide na camada oculta e uma função de ativação linear na saída.

Tabela I: Característica da Rede NARX

\begin{tabular}{|c|c|c|}
\hline $\begin{array}{c}\text { Números de } \\
\text { Camadas }\end{array}$ & $\begin{array}{c}\text { Número de } \\
\text { neurônios }\end{array}$ & $\begin{array}{c}\text { Número de } \\
\text { Atrasos }\end{array}$ \\
\hline 1 & $1: 10$ & 2 \\
\hline $\begin{array}{c}\text { Funções de } \\
\text { Transferência }\end{array}$ & $\begin{array}{c}\text { Camada } \\
\text { Oculta }\end{array}$ & $\begin{array}{c}\text { Camada } \\
\text { de saída }\end{array}$ \\
\hline & Sigmóide & Linear \\
\hline $\begin{array}{c}\text { Algoritmo de } \\
\text { Treinamento }\end{array}$ & Scaled Conjugate Gradient \\
\hline
\end{tabular}

\section{Dados Para VAlidação}

Para o desenvolvimento do trabalho foram utilizado sinais experimentais disponibilizados pela Universidade de Vigo (Espanha) [21]. As gravações foram realizadas no porto de Vigo (Espanha). O banco de dados é composto por 90 registros 
que representam sons de 11 categorias de embarcações, por exemplo, barcos de pesca, lanchas e balsas de passageiros, também são disponibilizados informações sobre aspectos técnicos das gravações e condições ambientais.

Para a aquisição dos sinais foi utilizado um conjunto de três hidrofones posicionados em diferentes profundidades, os hidrofones foram ancorados no fundo do porto e presos a uma boia submersa para garantir a verticalidade e uma boia de superfície para recuperação. A Fig. 10 ilustra o diagrama de aquisição dos sinais no porto de Vigo.

Para a obtenção dos sinais experimentais foram utilizados gravadores digitais acústicos com taxa de amostragem de $52.734 \mathrm{~Hz}$. O banco de dados disponibilizado por [21] é útil para o desenvolvimento e teste de aplicativos baseados no processamento de sons de embarcações.

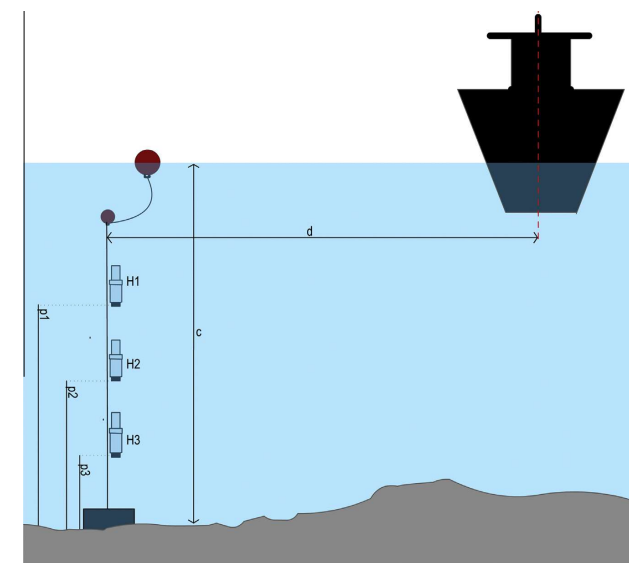

Figura 10: Sistema de aquisição de sinais acústicos submarinos do banco de dados ShipEar, adaptado de [21].

\section{Métricas de Validação}

Nessa seção, serão apresentadas as medidas de desempenho utilizadas para avaliar o simulador.

(1) O desvio percentual médio de frequência $\left(\bar{E}_{\text {har }}\right)$ é dado por (6) onde, $F_{q}$ e $F x_{q}$ são, respectivamente, o valor de frequência do q-ésimo harmônico $\left(q=1,2, \ldots, N_{h}\right)$ do sinal gerado pelo simulador e o do sinal experimental de referência;

$$
\bar{E}_{h a r}=\frac{1}{N_{h}} \sum_{q=1}^{N_{h}} \frac{\left|F_{q}-F x_{q}\right|}{F x_{q}} .100 \%
$$

(2) O erro absoluto médio $\bar{E}_{a m p}$ entre as amplitudes dos $N_{h}$ harmônicos do sinal do simulador $\left(A_{q}\right)$ e os do sinal de referência $\left(A x_{q}\right)$ é dado por (7):

$$
\bar{E}_{a m p}=\frac{1}{N_{h}} \sum_{q=1}^{N_{h}}\left|A_{q}-A x_{q}\right|[d B]
$$

(3) O erro entre os valores do nível médio de ruído de fundo do sinal do simulador fenomenológico $\left(A N L_{q}\right)$ e o do sinal experimental $\left(A N L_{x q}\right)$, é definido como $\Delta_{A N L}$ (8):

$$
\begin{gathered}
\Delta_{A N L}=\left|A N L_{q}-A N L_{x q}\right|[d B] \\
A N L_{q, x q}=\frac{1}{B} \sum_{k=1}^{B} A_{q, x q}[k][d B]
\end{gathered}
$$

em que:

$$
A_{q, x q}[k]=\left\{\begin{array}{l}
A_{q, x q}\left[k_{q}-\varepsilon\right], \text { para } k_{q}-\varepsilon<k \leq k_{q}+\varepsilon \\
A_{q, x q}[k], \text { caso contrário, }
\end{array}\right.
$$

$A_{q, x q}[k]$ é o espectro no k-ésimo índice de frequência discreta $(k=1,2, \ldots, B), B$ é o número de bins de frequência utilizado, $k_{q, x q}=F_{q, x q} / F_{s}$ é a frequência discreta dos $N_{h}$ componentes harmônicos $F_{q, x q}$ e $\varepsilon$ é o desvio de frequência em torno de cada $k_{q, x q}$, definindo a faixa em que os harmônicos são eliminados para o cálculo do valor $A N L_{q, x q}$. $\mathrm{O}$ valor de $\varepsilon$ foi fixado empiricamente.

\section{Resultados}

Nesta seção, inicialmente serão apresentados os resultados do simulador fenomenológico, seguido dos resultados relacionados a implementação da rede neural NARX. Para validação do simulador fenomenológico e treinamento da rede neural, foram usados sinais experimentais disponibilizado pela Universidade de Vigo (Espanha) [21].

O sinal usado como referência é oriundo de uma embarcação usada para transporte de passageiros chamada Mar de Onza, ver Fig. 11, e, no momento da aquisição dos dados, a embarcação estava saindo do porto de Vigo, o ruído estado do mar estava entre os níveis 0 e 1 e não estava chovendo.

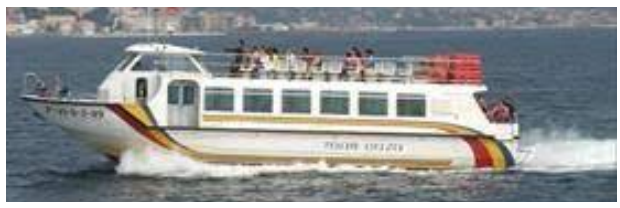

Figura 11: Embarcação para transporte de passageiros: Mar de Onza, adaptado de [23]

\section{A. Simulador Fenomenológico}

Inicialmente foi realizada uma análise DEMON do ruído experimental, ver Fig. 12, observa-se no demongrama há presença de três harmônicos, o primeiro harmônico $(847,7$ RPM) está relacionado à rotação do eixo do hélice da embarcação, os harmônicos seguintes (1701 e 2548 RPM) indicam a quantidade de pás do hélice. As informações obtidas (velocidade de rotação e n. $^{\circ}$ de pás) serão utilizadas como referência no simulador fenomenológico.

Para geração do ruído fenomenológico, foram inseridas no módulo de cavitação as seguintes informações: velocidade de rotação do eixo do hélice (primeiro harmônico) e a quantidade de pás do hélice (número de harmônicos), o módulo gerador de ruído ambiente foi configurado para simular o ruído estado do mar nível 0 , nível próximo do ruído ambiental na hora da aquisição do ruído experimental. 

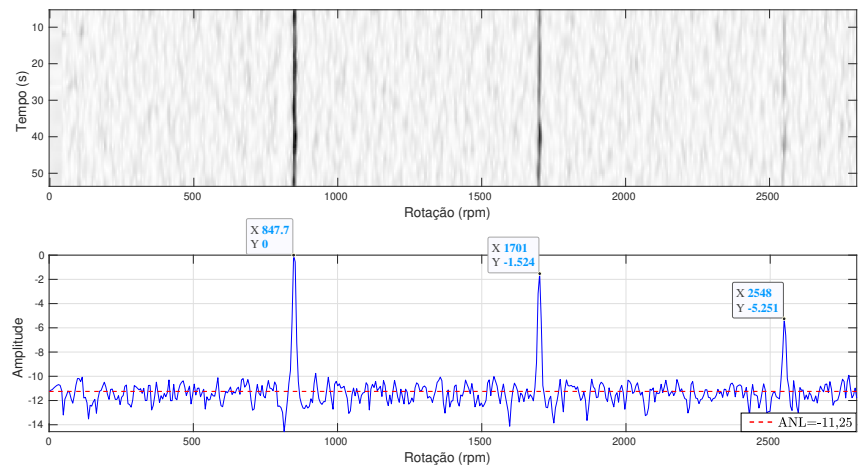

Figura 12: Análise DEMON do ruído experimental: embarcação Mar de Onza

O resultado é mostrado na Fig. 13. Comparando as Fig. 12 e 13, o $\bar{E}_{\text {har }}$ entre os picos dos harmônicos do sinal experimental e fenomenológico foi de $1.58 \%$ e o $\bar{E}_{a m p}$ foi de 2,480 $d B$, respectivamente. Para o erro médio entre os ruídos de fundo foi de $1,8 \mathrm{~dB}$.
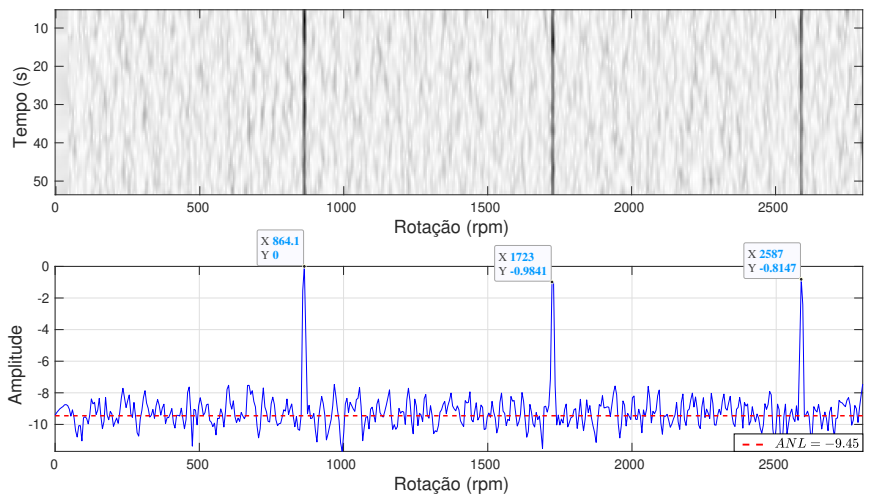

Figura 13: Análise DEMON do ruído fenomenológico

\section{B. Rede NARX}

A partir dos resultados apresentados na seção VI-A, percebe-se que o simulador fenomenológico consegue gerar um ruído de cavitação com comportamento espectral próximo do ruído experimental. No entanto, ele não consegue incorporar informações não-lineares ao ruído fenomenológico, como, por exemplo, a diferença de intensidade de cavitação de cada pá (intensidade dos harmônicos). Visando aproximar o simulador fenomenológico do ruído experimental, foi implementado uma rede neural NARX após o simulador fenomenológico.

Utilizando os ruídos do simulador fenomenológico como dados de entrada para a rede e o ruído experimental (embarcação MAR de ONZA) como sinal de referência, foi realizado o treinamento da rede. Para encontrar a melhor configuração da rede neural, foi feita uma variação do número de neurônios da camada oculta (entre 1 e 10 neurônios).

$\mathrm{O}$ treinamento que forneceu o melhor resultado foi a rede com configuração com 3 neurônios. Para configurações superiores não foi detectada melhorias no ruído, no entanto, houve um aumento no tempo de treino e uma maior exigência de processamento do computador. A Fig. 14 apresenta o decaimento do erro, o treino foi interrompido na interação 257.

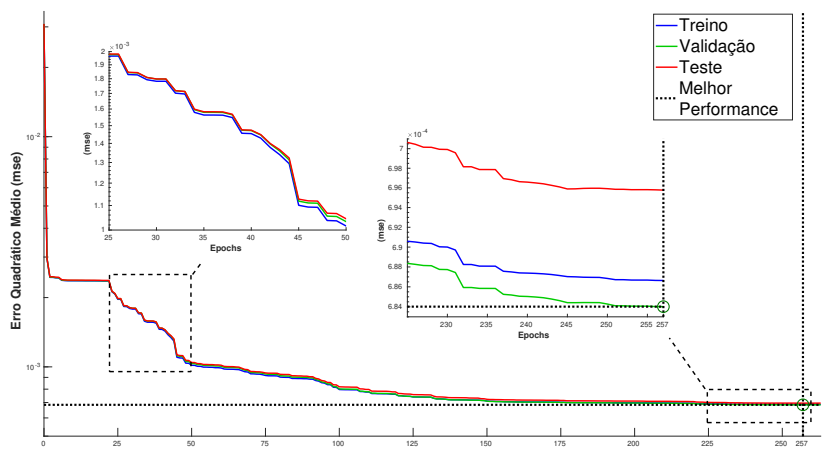

Figura 14: Gráfico do treino da rede NARX

A Fig. 15 apresenta a análise DEMON do ruído gerado no treinamento da rede contendo 3 neurônios. Observa-se que a rede NARX conseguiu aproximar o ruído fenomenológico do experimental, a velocidade de rotação do eixo do hélice e os valores dos harmônicos estão correspondendo ao do ruído experimental, no entanto, a intensidade dos harmônicos, apesar de se aproximarem, não estão iguais ao ruído experimental, o $\bar{E}_{a m p}$ foi de $0,01 d B$. O ruído médio de fundo, $A N L$ entre os dois ruídos está bem próximo, $\Delta_{A N L}=0,06$.
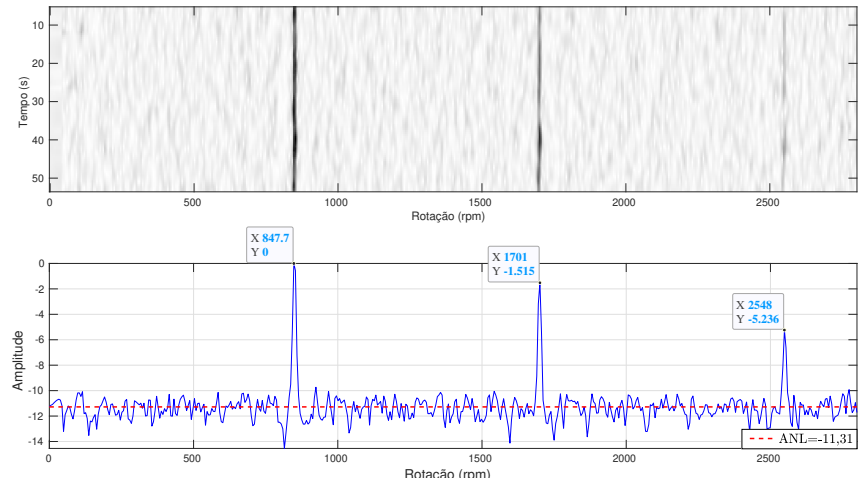

Figura 15: Ruído gerado no treinamento da rede NARX: Embarcação Mar de Onza

Após o treinamento, foi realizado uma avaliação das redes já treinadas (1 a 10 neurônios) utilizando um novo conjunto de dados. O intuito é avaliar o desempenho de cada rede, pois, após o treinamento, não haverá mais ajustes dos pesos, também não será fornecido um ruído de referência. As redes que forneceram melhor resultados no teste foram as redes com 2 e 9 neurônios, Fig. 16 e Fig. 17, respectivamente.

Comparando os resultados do teste das redes, ver Tabela II, observa-se que tanto a rede com 2 neurônios quanto a rede com 9 conseguiram melhorar o ruído gerado pelo simulador fenomenológico. A rede com 2 neurônios conseguiu um melhor desempenho da performance $\bar{E}_{a m p}$, enquanto a rede com 9 neurônios gerou melhores resultado no $\Delta_{A N L}$. Com relação 
a frequência dos harmônicos, nenhuma das redes apresentou melhoras.
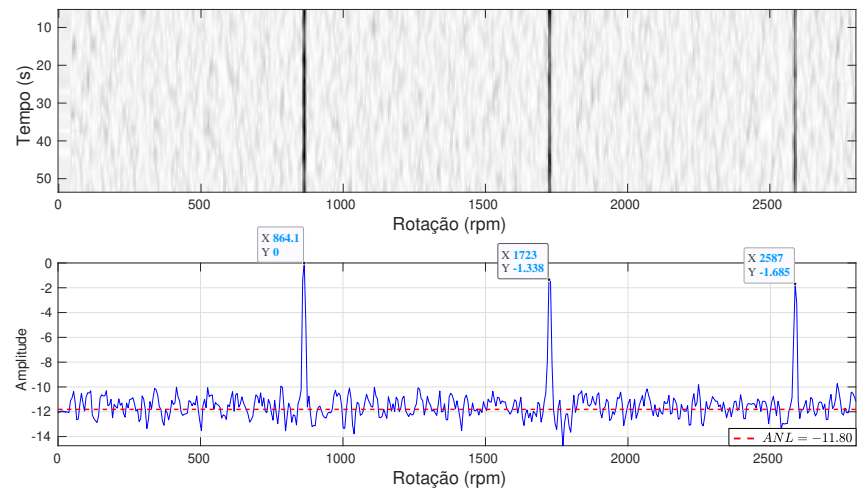

Figura 16: Sinal gerado pela rede com 2 neurônios:Embarcação Mar de Onza
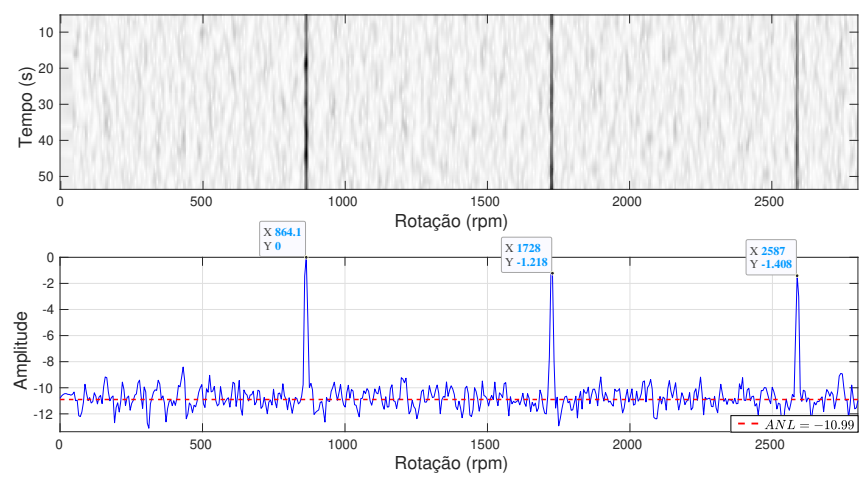

Figura 17: Sinal gerado pela rede com 9 neurônios: Embarcação Mar de Onza

Também foi feita análise do simulador de ruídos acústicos no domínio do tempo. Na Fig. 18, observou-se que a rede NARX consegue gerar um sinal mais próximo do sinal experimental do que o simulador fenomenológico.

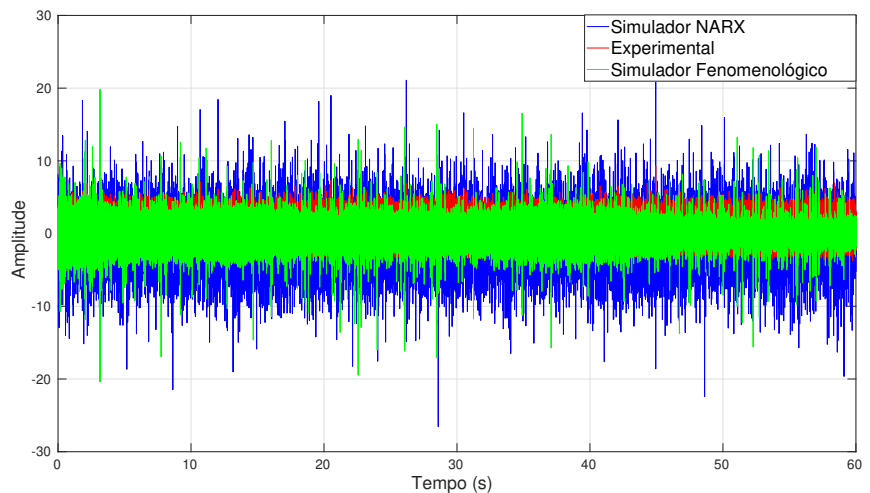

Figura 18: Sinais gerados pelo simulador no domínio do tempo da embarcação MAr de Onza

Com o intuito de verificar o desempenho da rede NARX para outros sinais experimentais, foi realizado o treinamento e teste da rede usando o sinal de outra embarcação, o sinal experimental pertence a um navio usado para o transporte de cargas (Eimskip Reefer). A Fig. 19 apresenta demongrama do Eimskip Reefer, observa-se no demongrama a presença de cinco harmônicos, o que indica a presença de um hélice com 5 pás e velocidade de rotação em 486,7 RPM.
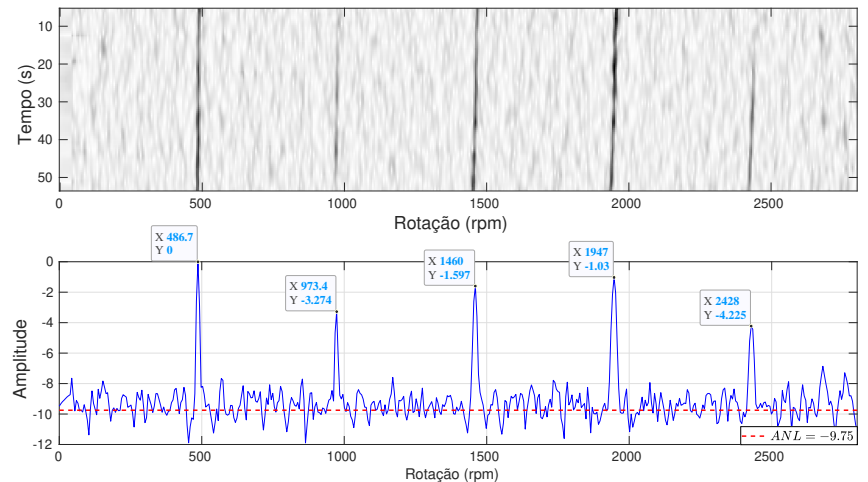

Figura 19: Análise DEMON da Eimskip Reefer

As Fig. 20 e 21 apresentam, respectivamente, a análise DEMON para os sinais gerados pelo simulador fenomenológico e rede NARX. A rede que gerou melhor resultado foi a rede com 9 neurônios na camada oculta. A Tabela II apresenta a comparação entre as análises de desempenho, nota-se que a rede NARX forneceu bons resultados para o ruído de fundo, $\Delta_{A N L}=0,23$, no entanto, a rede não conseguiu melhorar as frequências dos harmônicos.
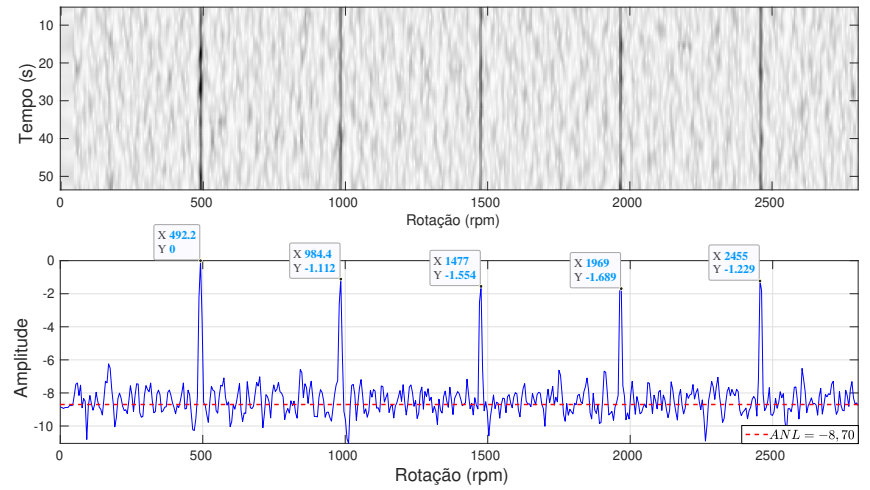

Figura 20: Ruído fenomenológico: Embarcação Eimskip Reefer

\section{CONCLUSÕES}

Este trabalho teve como objetivo desenvolver um sistema capaz de sintetizar ruídos acústicos submarinos. A princípio, utilizando o simulador fenomenológico, foi gerado um ruído a partir de informações de uma embarcação de transporte de passageiros. Os resultados obtidos pela análise DEMON mostraram que o simulador fenomenológico pode simular um ruído de cavitação com comportamento espectral próximo das especificações fornecidas ao simulador.

Em seguida, com o intuito de aproximar o ruído fenomenológico do ruído experimental, foi incorporado ao simulador uma rede neural NARX. A rede apresentou resultados satisfatórios ao tentar aproximar o ruído fenomenológico do expe- 

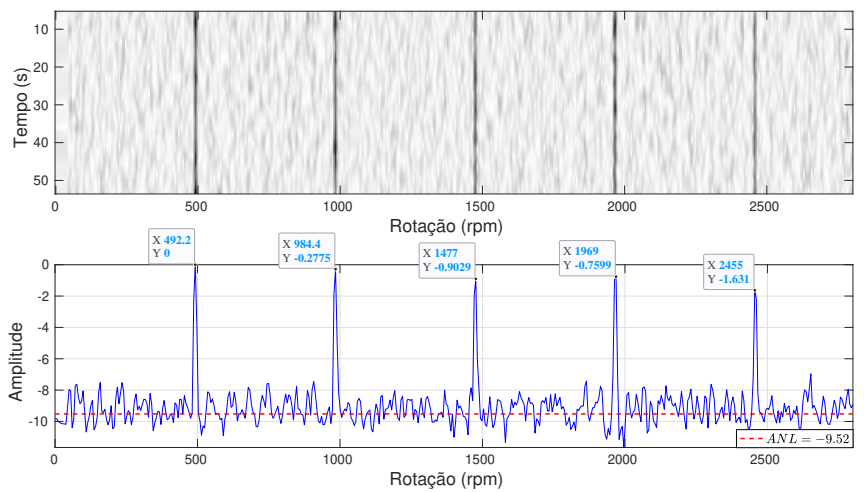

Figura 21: Sinal gerado ela rede com 9 neurônios: Eimskip Reefer

Tabela II: Comparação dos índices de desempenho entre o sinal experimental e o simulador

\begin{tabular}{|c|c|c|c|}
\hline $\begin{array}{c}\text { Embarcação } \\
\text { Mar de Onza }\end{array}$ & $\begin{array}{c}E_{\text {har }} \\
(\%)\end{array}$ & $\begin{array}{c}E_{a m p} \\
(d B)\end{array}$ & $\begin{array}{c}\Delta_{A N L} \\
(\mathrm{~dB})\end{array}$ \\
\hline \hline $\begin{array}{c}\text { Simulador } \\
\text { Fenomenológico }\end{array}$ & 1.58 & 2,48 & 1.8 \\
\hline $\begin{array}{c}\text { Treinamento da } \\
\text { Rede }\end{array}$ & 0 & 0,01 & 0,06 \\
\hline $\begin{array}{c}\text { Teste da rede c/ } \\
\text { 2 neurônios }\end{array}$ & 1,58 & 1,87 & 0,55 \\
\hline $\begin{array}{c}\text { Teste da rede c/ } \\
9 \text { neurônios }\end{array}$ & 1,68 & 2,07 & 0,26 \\
\hline \hline $\begin{array}{c}\text { Embarcação } \\
\text { Eimskip Reefer }\end{array}$ & $\begin{array}{c}E_{h a r} \\
(\%)\end{array}$ & $\begin{array}{c}E_{a m p} \\
(d B)\end{array}$ & $\begin{array}{c}\Delta_{A N L} \\
(\mathrm{~dB})\end{array}$ \\
\hline \hline $\begin{array}{c}\text { Simulador } \\
\text { Fenomenológico }\end{array}$ & 1.13 & 1.46 & 1.05 \\
\hline $\begin{array}{c}\text { Teste da rede cl } \\
9 \text { neurônios }\end{array}$ & 1,13 & 1,63 & 0,23 \\
\hline
\end{tabular}

rimental. Analisando os resultados vê-se que a rede NARX tem bom desempenho ao tentar aproximar o ruído banda larga, já para a banda estreita a rede encontrou dificuldades para melhorar o ruído fenomenológico. A rede também gerou bons resultados para o ruído no domínio do tempo. Uma das dificuldades encontradas ao trabalhar com a rede neural para aprimorar o simulador, foi a necessidade de maior processamento de dados. À medida que incrementamos a configuração da rede com o intuito de obter melhores resultados, maior foi a exigência de processamento.

Para trabalhos futuros, implementações de diferentes arquiteturas de aprendizado de máquinas, como, por exemplo, modelo LSTM (Long short-term memory) objetivando melhorar o simulador de ruído acústico, tando no domínio do tempo quanto da frequência, serão desenvolvidas.

\section{Agradecimentos}

O presente trabalho foi realizado com apoio da Coordenação de Aperfeiçoamento de Pessoal de Nível Superior - Brasil (CAPES) - Código de Financiamento 001. Os autores gostariam de agradecer também à FAPESB, FAPERJ e CNPq pelo apoio financeiro, e à Marinha do Brasil, por disponibilizar os dados experimentais e à Sonitum - AtlantTIC (Universidade de Vigo) pelo acesso ao conjunto de dados experimentais.

\section{REFERÊNCIAS}

[1] P. C. Etter, Underwater acoustic modeling and simulation. CRC press, 2018.

[2] F. Francisco and J. Sundberg, "Detection of visual signatures of marine mammals and fish within marine renewable energy farms using multibeam imaging sonar," Journal of Marine Science and Engineering, vol. 7, p. 22, Jan 2019

[3] G. Yu, D. Zhao, and S. Piao, "Target detection method using multipath information in an underwater waveguide environment," IET Radar Sonar \& Navigation, vol. 14, no. 2, pp. 226-232, 2020.

[4] B. Chen, Y. Yang, J. Zhou, Y. Zhuang, and M. McFarland, "Damage detection of underwater foundation of a chinese ancient stone arch bridge via sonar-based techniques," Measurement, vol. 169, p. 108283, 072020.

[5] R. P. Hodges, Underwater Acoustics Analysis, Design and Performance of SONAR. Reino Unido: John Wiley and Sons, Ltd, 2010.

[6] L. Bjørnø, T. Neighbors, and D. Bradley, Applied Underwater Acoustics: Leif Bjørnø, 022017.

[7] M. K. Pine, K. Nikolich, B. Martin, C. Morris, and F. Juanes, "Assessing auditory masking for management of underwater anthropogenic noise," The Journal of the Acoustical Society of America, vol. 147, no. 5, pp. 3408-3417, May 2020.

[8] G. Guillermo, D. Ponce, J. Telles, and C. Carpio, "An algorithm to obtain boat engine rpm from passive sonar signals based on demon processing and wavelets packets transform," Journal of Electrical Engineering \& Technology, vol. 14, 082019.

[9] N. N. de Moura, J. M. de Seixas, and R. Ramos, "Passive sonar signal detection and classification based on independent component analysis," Sonar Systems, InTech, pp. 93-103, 2011.

[10] Y. Wang, H. Li, and W. Dai, "Application of duffing oscillator in ship propeller blade number recognition," 2016, pp. 1-5.

[11] J. B. O. S. Filho, "Classificação neural de sinais de sonar passivo," Ph.D. dissertation, COPPE/UFRJ, 2007

[12] F. L. Santos, "Auxílio à classificação sonar passiva por tons característicos," Ph.D. dissertation, Universidade Federal do Rio de Janeiro COPPE-PEO, 2005

[13] N. Fisheries, "Ocean noise and soundscape projects," Mar 2021. [Online]. Available: https://www.fisheries.noaa.gov/ new-england-mid-atlantic/ocean-noise-and-soundscape-projects

[14] D. L. Bradley and R. Stern, Underwater sound and the marine mammal acoustic environment: A guide to fundamental principles. Bethesda, MD : Marine Mammal Commission,, 2008.

[15] R. M. de Amorim, "Avaliação da influência do ruído ambiente em sistema sonar passivo utilizando análise de componentes independentes," Master's thesis, Universidade Federal da Bahia - PPGEE/UFBA, Bahia, 2016.

[16] S. Haykin, Redes neurais: princípios e prática. Bookman Editora, 2001.

[17] Z. Yi, Convergence analysis of recurrent neural networks. Springer Science \& Business Media, 2013, vol. 13.

[18] Z. Boussaada, O. Curea, A. Remaci, H. Camblong, and N. M. Bellaaj, "A nonlinear autoregressive exogenous (narx) neural network model for the prediction of the daily direct solar radiation," Energies, vol. 11, no. 3, p. 620, 2018.

[19] S. Minfen, L. Sun, and F. H. Y. Chan, "Generation of noise sequences with desired non-gaussian distribution, and covariance," 2002 IEEE Region 10 Conference on Computers, Communications, Control and Power Engineering. TENCOM '02. Proceedings., vol. 2, pp. 1105-1108, 2002.

[20] Z. Zhiu and Z. Yong-sheng, "Research on the underwater vehicle radiated noise model and the linear spectrum extraction method," 2016 13th International Conference on Ubiquitous Robots and Ambient Intelligence (URAI), pp. 819-823, 2016.

[21] D. Santos-Domínguez, S. Torres-Guijarro, A. Cardenal-López, and A. Pena-Gimenez, "Shipsear: An underwater vessel noise database," Applied Acoustics, vol. 113, pp. 64-69, 2016.

[22] D. Nguyen and B. Widrow, "Improving the learning speed of 2-layer neural networks by choosing initial values of the adaptive weights," 1990 IJCNN International Joint Conference on Neural Networks, pp. 21-26, 1990.

[23] "Vessel mar onza, vessel details and voyage information - registered in spain." [Online]. Available: https://www.marinetraffic.com/en/ais/details/ ships/shipid:164118/mmsi:224081370/imo:0/vessel:MAR_ONZA 